\title{
Condition-based diagnosis of mechatronic systems using a fractional calculus approach
}

\author{
Ricardo Enrique Gutie'rrez-Carvajal, Leonimer Flávio de Melo, João Maur'́cio Rosário and J.A. TenreiroMachado
}

\begin{abstract}
While fractional calculus (FC) is as old as integer calculus, its application has been mainly restricted to mathematics. However, many real systems are better described using FC equations than with integer models. FC is a suitable tool for describing systems characterised by their fractal nature, long-term memory and chaotic behaviour. It is a promising methodology for failure analysis and modelling, since the behaviour of a failing system depends on factors that increase the model's complexity. This paper explores the proficiency of FC in modelling complex behaviour by tuning only a few parameters. This work proposes a novel two-step strategy for diagnosis, first modelling common failure conditions and, second, by comparing these models with real machine signals and using the difference to feed a computational classifier. Our proposal is validated using an electrical motor coupled with a mechanical gear reducer.
\end{abstract}

Keywords: intelligent maintenance; intelligent diagnostics; application of fractional calculus; identification of fractional order systems

\section{Introduction}

Competing in the global market requires the production of high-quality goods with low development and manufac- turing periods. New strategies that result in a faster qual- ity control of manufactured products, whilst minimising downtime due to equipment maintenance, are thus essen- tial (Gonc,alves, 2011; Liu \& Makis, 2008; Shikari \& Sadi- wala, 2004). A short product development time and the integration of several technologies require highly trained personnel to carry out traditional inspections, quality con- trols and fault diagnoses. Recently, there has been the de- mand for analytical techniques in signal-issued sensors, to describe the behaviour of devices with various compo- nents interacting. This corresponds to a demand for a more highly skilled workforce with a deep knowledge of differ- ent technologies to be used, supporting their diagnoses with computer-recommended systems.

A typical condition-based diagnosis system requires a set of signals with information concerning the current state of the machine, reflecting various phenomena such as vi- bration, noise, temperature and lubrication, amongst others (Funk \& Jackson, 2005; Jayaswal, Wadhwani, \& Mulchan- dani, 2008). Each signal needs to be treated while discarding irrelevant information accordingly to the type of failure to be isolated. The resulting signals are analysed by means of one or more processing techniques, in order to simplify the failure detection process. That set of signals must contain sufficient information to identify the machine's condition, allowing an expert to diagnose the device and plan a maintenance action (Bengtsson, Olsson, \& Funk, 2004).

A common computer-aided technique used in fault diagnosis identifies the dynamic system of a machine using ordinary differential equations (Duvar, Eldem, \& Saravanan, 1990). In the presence of a fault, the system leads to the variation of specific parameters, useful not only to diagnose the problem, but also to estimate the state of the failure. However, this strategy is only useful when the de- vice is simple and its model can be satisfactorily identified adopting a reduced number of parameters (Ljung, 1987). In this case, the space of parameters is small enough to neglect the problem of dimensionality (Kantardzic, 2003). Unfor- tunately, this is not a common situation as real systems typically contain a large number of interactive components, as well as phenomena that are difficult to model (Wang, Wang, \& Han, 2010).

Fractional calculus (FC) has been applied by researchers from different areas, due of its ability to describe complex phenomena using a smaller number of parameters, than its integer counterpart, so as to say, taking advantage of the additional degree of freedom given by the arbitrary order (Espindola, Bavastri, \& Lopes, 2008; Gutie'rrez-Carvajal, Rosário, \& Machado, 2010; Hartley \& Lorenzo, 2003). 
However, its use has been restricted to some engineering problems (Santos, Silva, \& Suetake, 2012), mainly due to the lack of a simple geometric and physical interpretation (Machado, 2013; Podlubny, 1994). In practice, the solution of a dynamic model of fractional order approximates com-

plex behaviours emerging from systems with multiple interactions (Vinagre, 2007). Consequently, many real systems can be better approximated using compact fractional order equations (Petras, 2006). This is a desirable approach to an automatic system for failure identification, since automatic classifiers require a balance between informative inputs and the amount of entries (Kantardzic 2011 PCTherefore iden-et of indicators

of the machine's condition associated with each parameter. This work proposes a new methodology based on intelligent

maintenance that continually assesses the condition of the system, restricting the amount of required parameters in the identification process. The identified model is used in a classification algorithm that allows the device to be diagnosed.

The paper is organised as follows: Section 2 introduces the fundamentals of FC; Section 3 demonstrates the ex- perimental workbench configuration; Section 4 presents FC implementation and the system validation diagnostic; Section 5 illustrates some experimental results and Sec- tion 7 outlines the main conclusions of this work.

\section{Fundamentals of fractional calculus}

FC represents the generalisation of integer calculus to real or complex order (Adams, Hartley, \& Lorenzo, 2006). One of the reasons why derivatives and integrals of fractional order are still relatively unknown in engineering is that the calculation of fractional order has multiple definitions (Ortigueira, Machado, \& da Costa, 2005), making it diffi- cult to interpret geometrically (Machado, 2003; Moshrefi- Torbaty \& Hammond, 1998; Podlubny, 2002). However, many phenomena are described by formulations of frac- tional order, as it has the ability to express the past behaviour by means of a limited number of coefficients (Magin \& Ovadia, 2008; Machado, Galhano, \& Trujillo, 2014). In this work, we use the Riemman-Liouville formulation, whose integral $\left(J_{C}\right)$ and derivative $\left(D^{\alpha}\right)$ definitions are introduced in the following equations, respectively (Cafagna, 2007):

$J^{\alpha}$

$$
\begin{gathered}
J_{c}^{\alpha} f(t)=\frac{1}{\Gamma(\alpha)} \int_{c}^{t} \frac{f(\tau)}{(t-\tau)^{1-\alpha}} d \tau, \\
D^{\alpha} f(t)=\frac{d^{m}}{d t^{m}}\left[\frac{1}{\Gamma(m-\alpha)} \int_{0}^{t} \frac{f(\tau)}{(t-\tau)^{\alpha+1-m}} d \tau\right],
\end{gathered}
$$

with $m \in \mathrm{Z}^{+}$and $m-1<\alpha \leq m$. The symbol $r$ stands for gamma function (Gorenflo \& Mainardi, 2008; Vale'́rio,

Trujillo, Rivero, Machado, \& Baleanu, 2013), defined as

$$
\Gamma(x) \equiv \int_{0}^{\infty} y^{x-1} e^{-y} d y,
$$

or

$$
\begin{gathered}
\Gamma(x) \equiv \lim _{N \rightarrow \infty}\left[\frac{N ! N^{x}}{x(x+1)(x+2) \ldots(x+N)}\right], \\
\forall x \notin \mathbb{Z}^{-} \cup 0 .
\end{gathered}
$$

An advantage of using this definition is that, unlike other definitions, it has a strict definition of the Laplace transformation, which facilitates identification algorithms.

It is formally written as

$$
\begin{aligned}
& \left.\qquad \mathcal{L}\left\{_{0} D_{t}^{\alpha} f(t)\right\}=s^{\alpha} F(s)-\sum_{j=0}^{n-1} s^{j}{ }_{0} D^{\alpha-j-1} f(0)\right], \quad \text { (5) } \quad L\left\{0 D^{\alpha}\right. \\
& \text { with } n-1<q<n \text { and } n \in \mathbb{Z} \text { (Ma \& Hori, 2004; Valério } \\
& \text { et al., 2013). }
\end{aligned}
$$

\section{Experimental workbench configuration}

This section defines the experimental workbench proposed for testing and validation. Our experimental bench for test- ing the fault-detection algorithm proposed herein consists of transmitting power through a gear reduction with mul- tiple stages driven by a DC motor, as shown in Figure 1. An accurate model of this machine is difficult to obtain analytically, due to the large number of interactive compo- nents such as gears and bearings. Moreover, the interaction between two gears is still not a well-known phenomenon, with many parameters difficult to measure or to estimate, since they depend on imperfections in the surface of the teeth, the shape of the profile, the contact time between teeth, temperature, friction and others.

The test bench include a voltage source which feeds actuators and instrumentation equipment, a DC motor to drive the gearbox composed of four gears produced by rapid pro- totyping which introduces several types of faults in a simple way. An accelerometer measures the bearing vibration, as shown in Figure 2. Signals generated by the actuator are measured by a resistor (motor current) and a tachometer (speed of the motor shaft). The signals from sensors are sent to a central computer through the data acquisition interface PCI-6221 of National Instruments $\mathbf{Q}$, with a CB-68LP card. The set of acquisition has 16 -bit precision in reading ana-

logue signals and a maximum sampling frequency of 2.5

MHz. The acquired signals are stored in a database generated by a control programme acquisition implemented in Labview $\mathbf{Q}$.

Figure 3 shows the workbench operation diagram. It $i$ not possible to measure a fault directly from the source, due to the transmission path to be physically followed before the signal is measured. Figure 2 demonstrates the trans- mission path between a localised failure in the fourth gear, the accelerometer (vibration sensor) and the resistor (motor current sensor). 


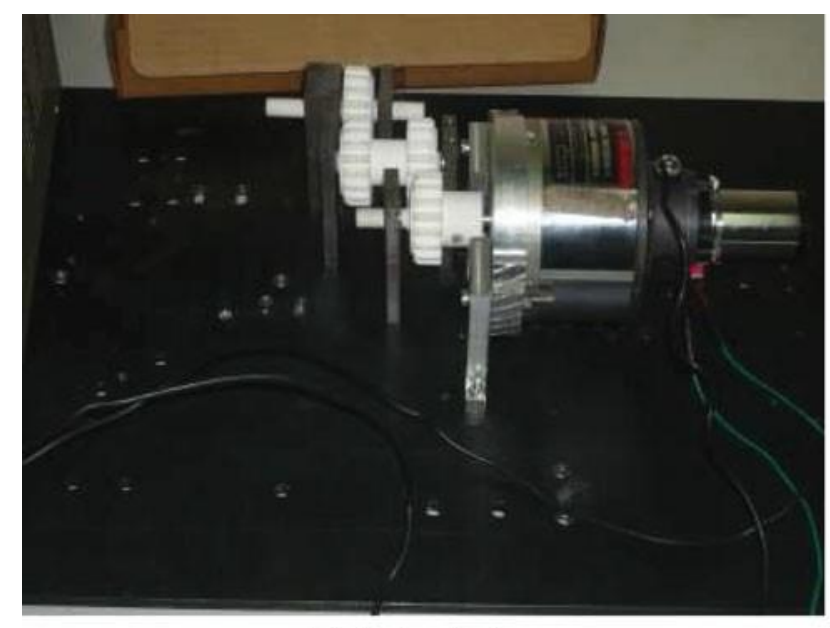

(a) Lateral view

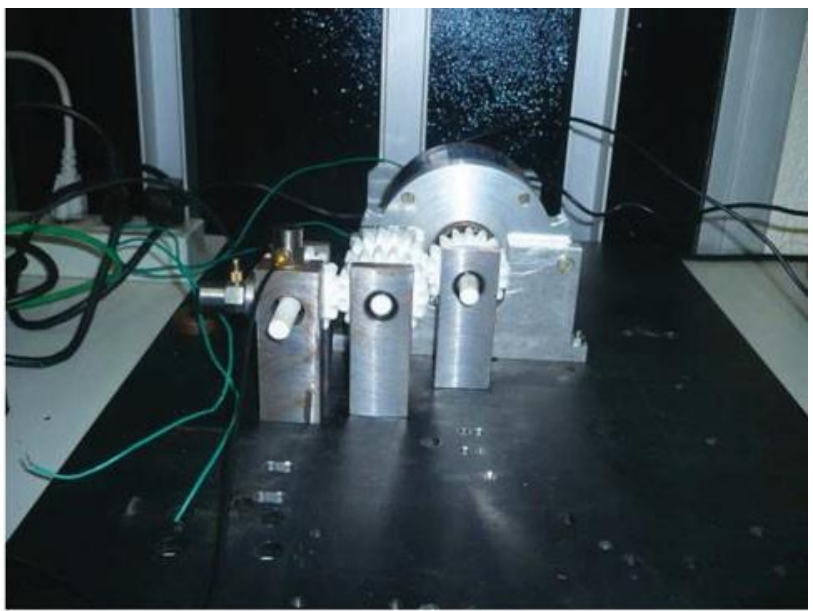

(b) Frontal view

Figure 1. Experimental platform.

This work studies four operating conditions, corre- sponding to the transmission path from the failure until the sensor, as follows:

- Case 1. Normal operating conditions. The system shows no fault and operates as standard.

- Case 2. Broken tooth in gear 2. The second gear is missing one of its nine teeth. The vibration signal (related to the failure) propagates through gears 2, 3 and 4 , up to being measured by the accelerometer. This is a failure that least affects the signal obtained by the accelerometer, since the long transmission path reduces its intensity. Moreover, the signal propagates through the resistor in the circuit of the motor current.

- Case 3. Broken tooth in gear 3. The pattern of vibrationrelated failure in gear 3 modulates through

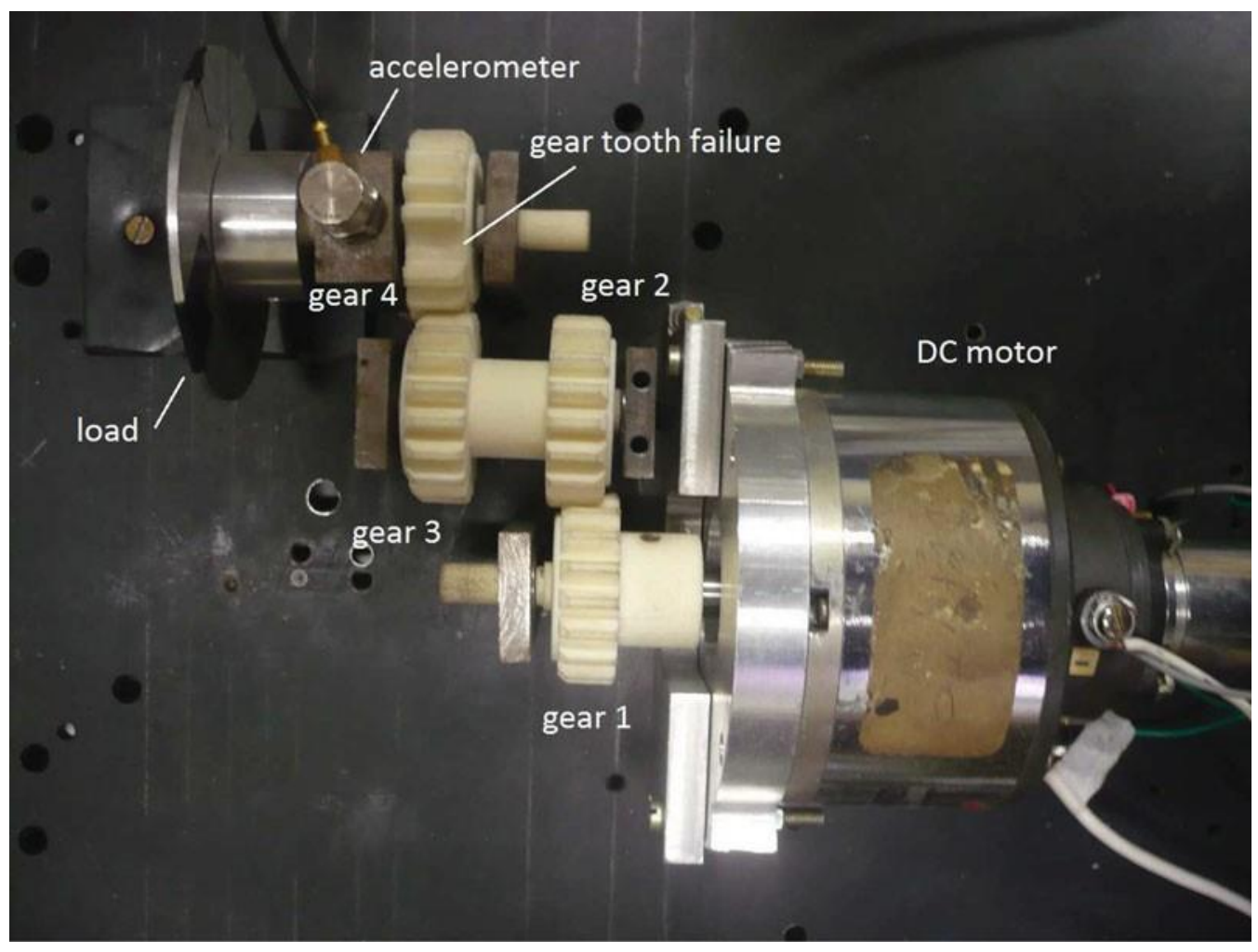

Figure 2. Detail of the gearbox and transmission path associated with failure of one tooth in gear 4 . 


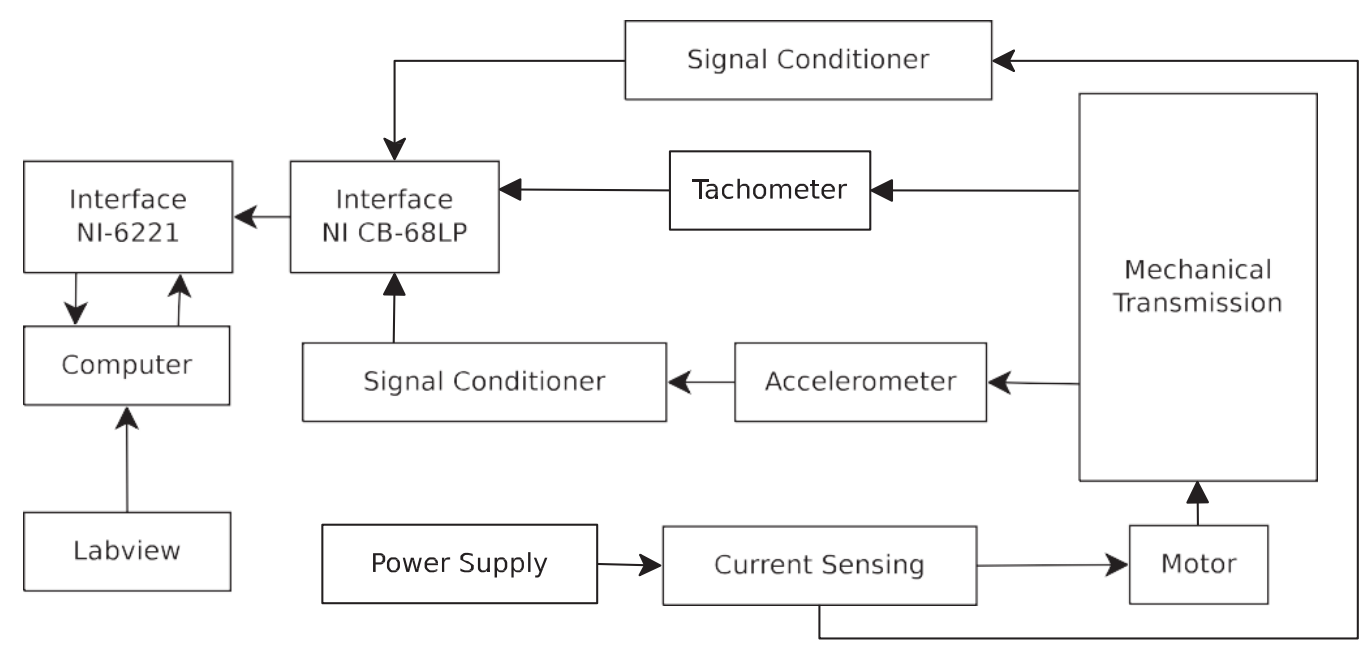

Figure 3. Block diagram of the experimental bench.

gears 3 and 4 (transmission path to the accelerom- eter). The fault signal (which affects the motor cur- rent) propagates through gears $1,2,3$, and the motor circuit.

- Case 4. Broken tooth in gear 4. With the failure of missing a cog, the transmission path of the signal propagates through gear 4 to reach the accelerometer. Moreover, propagation takes place by gears 1,2, 3, and the motor circuit.

\section{FC implementation and system validation diagnostic}

The methodology for the development of the proposed strategy is based on the layered model, Open Systems Architecture for Condition-Based Maintenance (OSA-CBM) (Bengtsson et al., 2004). Initially, different signals of in- terest were acquired to diagnose failures such as vibra- tion, position, electric current and others (Layers 1 and 2, OSA-CBM). The filtering is performed with the compo- nents of the acquired signal and by identifying the model parameters (Layer 3). These operations generate a set of indices, which allow the state of the machine (Layer 4) to be assessed, before being diagnosed by an experienced worker, or by a system with some diagnosis analysis tech- nique (Layer 5).

\subsection{Adjusting the system gear model}

Identify a model in the following four differentsteps:

(1) Assume a model structure to be identified.

(2) Obtain and process the experimental data.

(3) Identify the model parameters.

(4) Validate the model by comparing the results to a set of data that was not used to find the parameters.
Consequently, identifying a system can be treated as a problem of minimising the error obtained between the model and the actual data. Therefore, one must find the best

parameter vector pethat minimises the objective function

error $f_{e}$ from the real system $G r(s)$ and the $\operatorname{model} G m(\boldsymbol{p}, s)$ :

$$
f_{e}(\vec{p})=\left|G_{r}(s)-G_{m}(\vec{p}, s)\right| .
$$

Different approaches may be used to minimise (6) with- out losing generality. In this study, we adopt the simplex method (Lagarias, Reeds, Wright, \& Wright, 1998), which consists of an iterative algorithm that searches for a candi- date solution by calculating the centroid from three starting points. It subsequently analysed whether the centroid is bet- ter than any of the starting points, and, if so, it replaces the worst of them. The algorithm runs until it converges, or until it reaches a specified number of iterations.

\subsection{Objective function}

The input signals are obtained from an accelerometer lo- cated over the output bearing, since it must obtain the vibration due to failure. Changes on the vibration signa- tures directly affect the motor torque and hence the motor current (output signal). For each failure mode presented herein, information was acquired from the experimental bench working at different speeds. After obtaining accel- eration records, a filtering operation was applied using a moving average (MA) to reduce the effect of noise. Fur- thermore, the Fourier transformation was calculated using the Hanning window with a duration of one second to re- duce noise introduced during the scanning process. Here the motor current $(I)$ is considered as the system output and the voltage generated by the accelerometer as input $(V)$. We can define the current state of the device with the empirical estimation of the transfer function (EETF) (Ljung, 1987) 
as follows:

$$
G_{\mathrm{EETF}_{i}}(\omega)=\frac{\mathcal{F}\left\{I_{i}(t)\right\}}{\mathcal{F}\left\{V_{i}(t)\right\}} \quad \text { where } i \in\{1,2,3,4\}, \quad \text { (7) } \begin{aligned}
& \text { where } \\
& F(\cdot) \text { is } \\
& \text { the }
\end{aligned}
$$

Fourier transform, $i$ denotes the $i$ th failure and $\omega$ is the angular frequency, in the range $100-1000 \mathrm{rad} / \mathrm{s}$, since the beat frequency of the gears' teeth is within that

bandwidth.

We propose identifying each EETF $i$ using an FC model, with a structure having five parameters:

$$
G_{i}(s)=\frac{1}{a s^{\alpha}+b s^{\beta}+c}, \quad\{a, b, c, \alpha, \beta\} \in \Re
$$

The parameters of this model were adjusted using a set of 20 data-sets for tuning and 10 data-sets for evaluation, with an objective function that minimises the error between $G \mathrm{EETF}_{i}$ and $g_{i}$ :

$$
f_{e}=\left|G_{\mathrm{EETF}_{i}}(\omega)-G_{i}(\omega,[a, b, c, \alpha, \beta])\right| .
$$

\section{We}

assume that a particular failure behaves close to a specific condition model. Therefore, we compute the dif- ference between the actual machine system $G$ EETF and each condition model $G i$, using the mean square error to each model as a failure index to be assessed by an automated classification system.

\subsection{Failure diagnosis}

The aim is to evaluate the proposed strategy for failure diagnosis. Our technique enables automated grading testing when a particular device is failing, and allows one to lo- cate the part with the problem and to assess its state. Faults are classified by the $k N N$ algorithm (k-nearest neighbours). This strategy involves comparing the model identified in the current state of the machine with a database contain- ing known flaws identified with models. The classification completes itself with the categories of $k$ closest systems, by means of a strategy of choice (Cover \& Hart, 1967). This method is presented in Algorithm 1.

Algorithm 1: $k$-nearest neighbours, where the type of failure is estimated from the more representatives in the $k$-neighbourhood

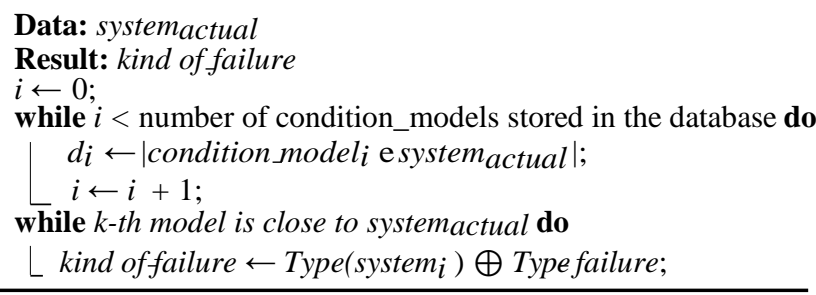

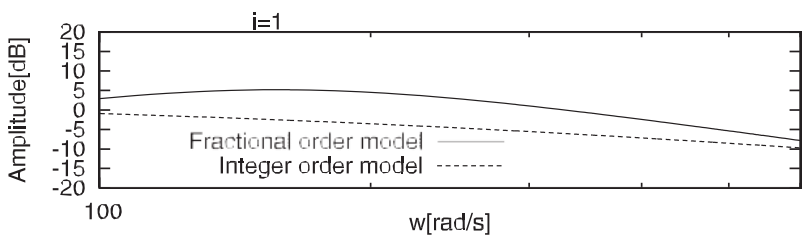

(a) Case 1.

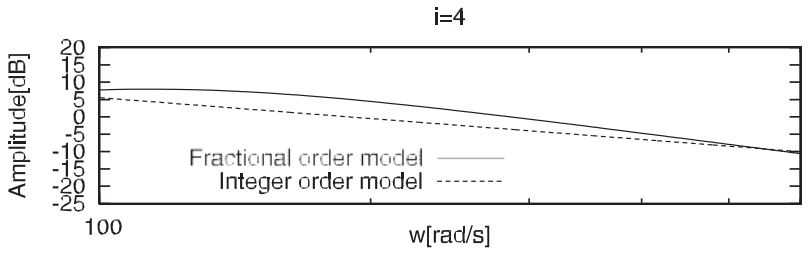

(b) Case 2 .

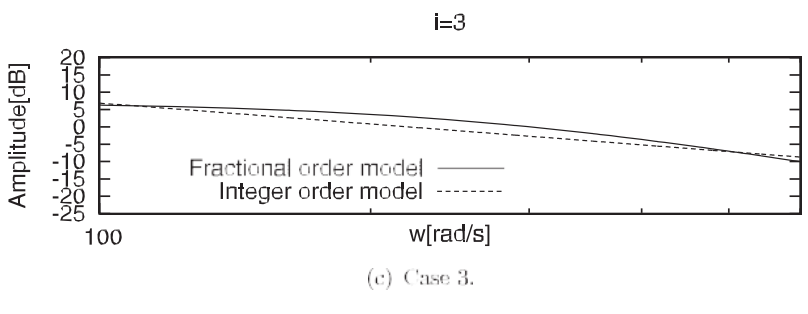

$\mathrm{i}=2$

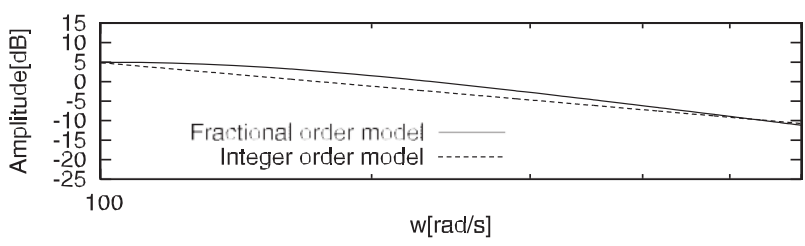

(d) Case 4

Figure 4. Approximation model (fractional and integer order) for each EETF failure.

\section{Results}

Following the methodology described, we adopted a set of 240 GEETF data records, not used previously for parameter identification of standard $G_{i}(s)$, introducing four known fault conditions. Figure 4 demonstrates the identification results using the proposed strategy; the results with the fractional order model (FOM) are also compared with a classical second integer order model (IOM), i.e. having

$\alpha=2$ and $\beta=1$.

Note that the fractional order approximation is consistent with the data and also more accurate than the integer approach. Table 1 depicts the average error and standard deviation, both for the data used for tuning the model and the data used for testing purposes. The FOM fits betterfor the whole data-sets than the IOM.

\subsection{Failure identification}

In order to test the generality of the technique, we adopted a strategy of rating 10 subsets, using one as a test set and 

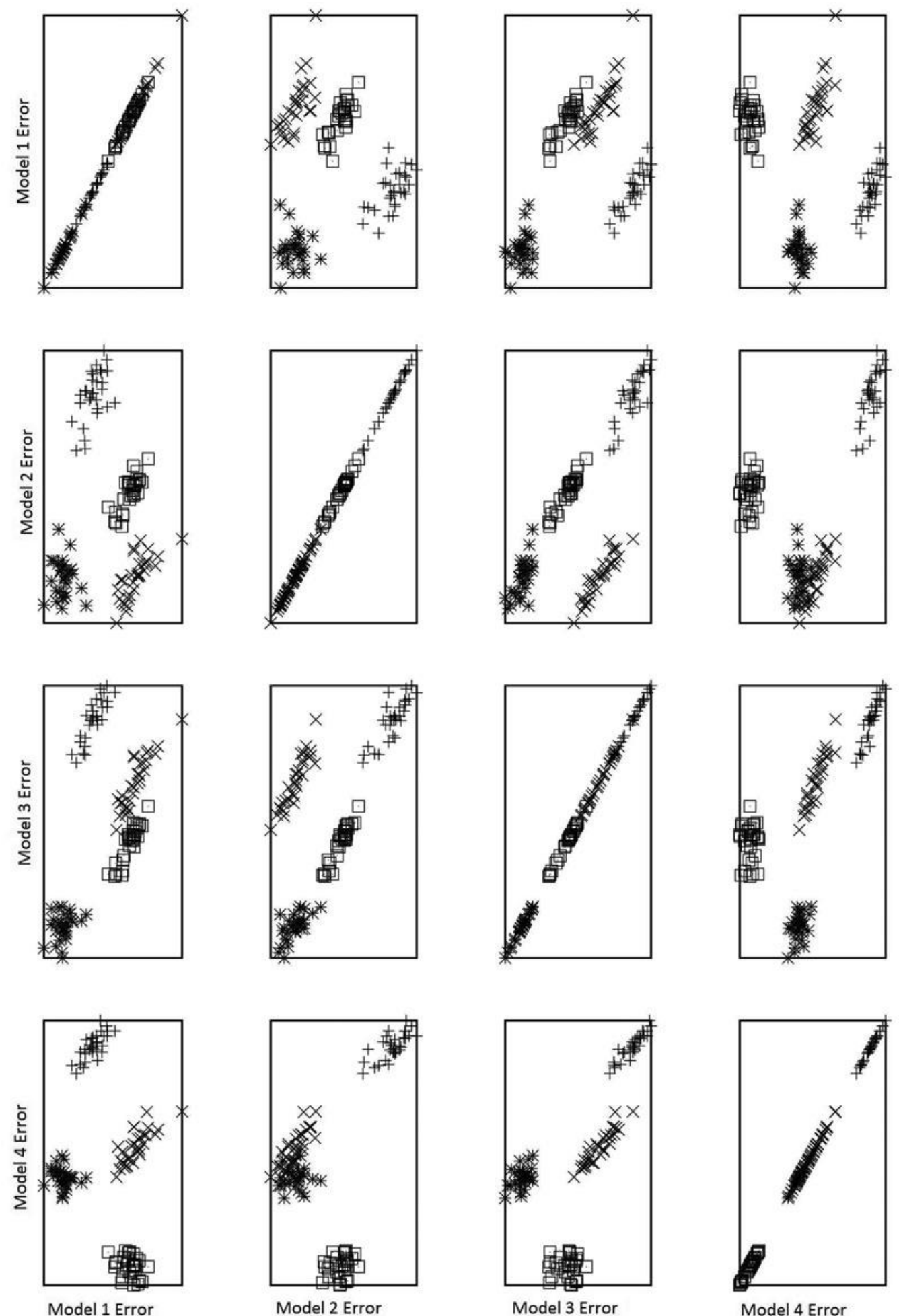

+ Case $1 \times$ Case 2

* Case $3 \square$ Case 4

Figure 5. Indices found during failure diagnosis. 
Table 1. Train and test mean errors \pm standard deviation of the FOM and the IOM when compared with real data.

\begin{tabular}{cccccc}
\hline & \multicolumn{2}{c}{ Train error \pm deviation } & & \multicolumn{2}{c}{ Test error \pm deviation } \\
\cline { 2 - 3 } \cline { 5 - 6 } & FOM & IOM & & FOM & IOM \\
\hline Case 1 & $1.84 \pm 0.15$ & $3.23 \pm 0.18$ & & $2.06 \pm 0.23$ & $3.47 \pm 0.27$ \\
& & & & & \\
Case 2 & $1.12 \pm 0.05$ & $2.26 \pm 0.05$ & & $1.05 \pm 0.02$ & $2.17 \pm 0.03$ \\
Case 3 & $1.44 \pm 0.34$ & $2.43 \pm 0.38$ & & $1.41 \pm 0.09$ & $2.37 \pm 0.10$ \\
Case 4 & $0.74 \pm 0.05$ & $1.36 \pm 0.06$ & & $0.89 \pm 0.07$ & $1.51 \pm 0.07$ \\
\hline
\end{tabular}

nine as training sets. We employed the use of $k N N$ here to estimate automatically a diagnostic, varying the number of neighbours $k$. The next step was to constitute another test set, repeating this operation until all data had been tested. Considering the results obtained during the estimation of the FOM, 60 GEETF samples were randomly taken for each case of study, with the aim of testing a classification strategy. The indices obtained are depicted in Figure 5. Note that, as expected, failure types are grouped into different spatial regions making it possible to use a very simple classification technique for equipment failure diagnosis.

On average, the classifier obtained similar performances independently of the neighbourhood size. Nevertheless, the lower data dispersion was achieved using three neighbours, as shown in Figure 6. Table 2 presents the results obtained with three neighbours.

\section{Discussion}

Nowadays, it is of paramount importance to quickly evalu- ate machinery and product performance in order to improve client services. A large part of failures occur due to wear on specific pieces of machinery. In fact, many maintenance procedures are planned, based on supplier requirements

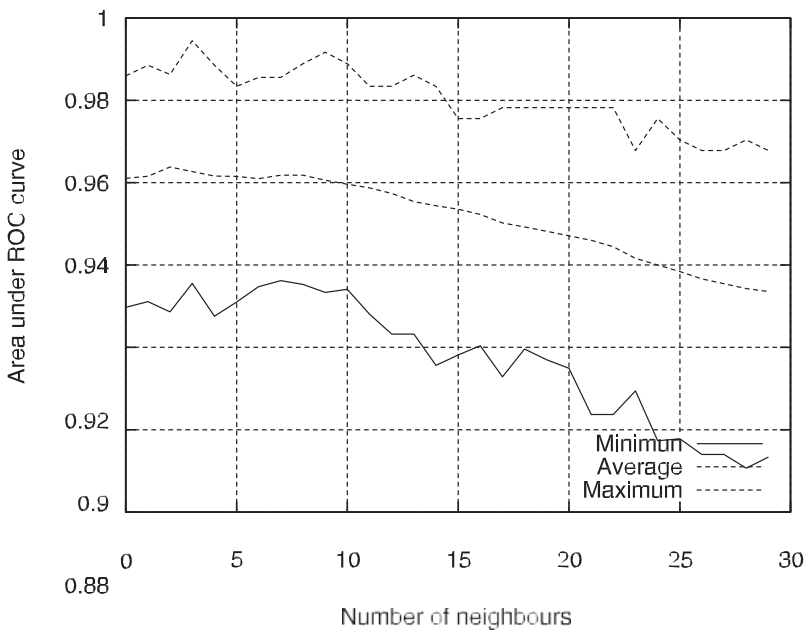

(a) Area under the ROC curve.
Table 2. Diagnosis estimation yielded by the proposed algorithm.

Estimated diagnosis

Case $1 \quad$ Case 2 Case $3 \quad$ Case 4

\begin{tabular}{lrrrrr}
\hline & Case 1 & 56 & 4 & 0 & 0 \\
Actual failure & Case 2 & 0 & 48 & 12 & 0 \\
& Case 3 & 0 & 0 & 60 & 0 \\
& Case 4 & 0 & 4 & 0 & 56 \\
\hline
\end{tabular}

(Endrenyi et al., 2001), due to the existence of a small set of known failures exist that affects machine performance. These failures could occur before or after a maintenance task, unnecessarily stopping the machine in the first case or, eventually causing a fatal failure. Herein, we analysed four particular machine conditions, being the normal per-formance point and three conditions of failure, all affecting different parts of the machine and measured using the same set of sensors. Other cases and degrees of failure could be considered using the same approach without losing gen- erality, that is, considering them as new conditions of the machine. For this set of failures, the system accurately di- agnoses the location of thefailure.

The algorithm proposed requires two main conditions: first, a general, but accurate, model of the system and, sec- ond, a known set of frequent failures to identify. In order to meet the first condition, we compared integer and fractional order models. The results reveal that FOM consistently ob- tains a better system than the IOM. It allows the algorithm to finally conform disaggregated groups, as presented in Figure 5, where each group represents a condition of the machine. This improved signal representation is due to the derivative operator that adds additional degrees of freedom. Conforming the groups allows a simple technique, suchas

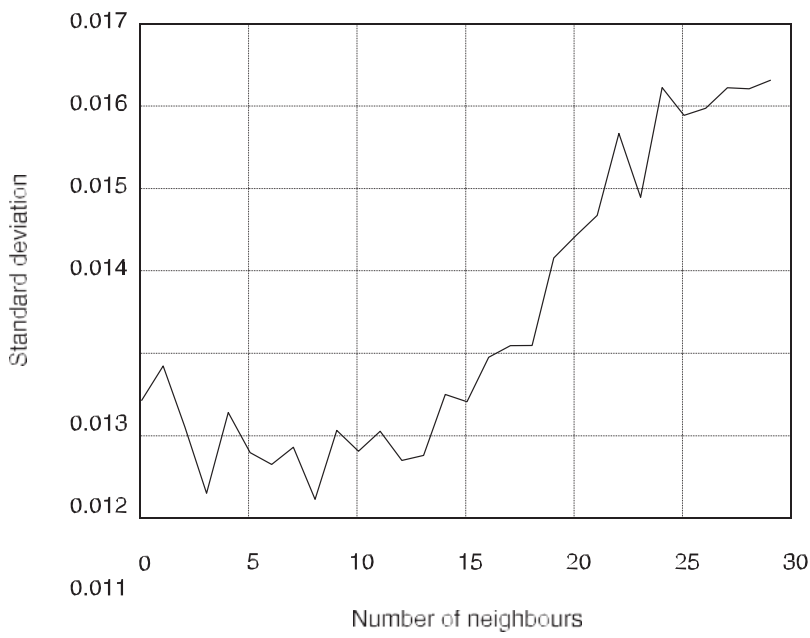

(b) Standard deviation of ROC values.

Figure 6. Effect of increase the number of neighbours $k$. 
$k N N$, to accurately diagnose the current condition of the machine. The amount of neighbours taken into account for voting to identify the machine's condition, does affect the classifier's performance, reducing the accuracy whilst in- creasing the uncertainty of the result, as shown in Figure 6.

\section{Conclusions}

FC is used in several scientific areas, but up to now, there have been no studies on the use of its adoption for fault prediction in the literature. However, fractional order al- gorithms are a promising tool for this type of modelling, since the system behaviour depends on the machine's op- eration history and the wear of the parts. Starting from a model close to the plant, it was possible to extract simple failure rates that are good descriptors of the current state of the device. Due to this fact, it was possible to use simple classification techniques proposed in the literature.

If we consider industrial requirements, $\mathrm{FC}$ is an alter- native strategy to obtain the state of a device, by means of identification systems based on the fact that a particular fault recurs quite frequently. These failures vary the opera- tion of the system in a known manner, which could also be identified using a model with few parameters. The proposed strategy generates a failure rate, in the frequency domain, that can be used to diagnose a particular device. The high accuracy of the implemented system in diagnosing failures is basically due to the use of a fractional order structure as the basis of the identification system. In fact, the use of a single canonical structure, using only three coefficients and two orders, was able to sufficiently approximate the device's behaviour for each failure under study.

\section{Acknowledgements}

The authors acknowledge the Campinas State University UNICAMP (Brazil), Polytechnic Institute of Porto - ISEP (Portugal) and the National Council for Scientific and Technological Development $-\mathrm{CNPq}$ (Brazil), for financially supporting this work, and also to Marian Lee for her help in proofreading this article.

\section{References}

Adams, J.L., Hartley, T.T., \& Lorenzo, C.F. (2006). Fractionalorder system identification using complex order-distributions. In Proceedings of the 2nd IFAC Workshop on Fractional Differentiation and its Applications, 2(1),345-356.

Bengtsson, M., Olsson, E., \& Funk, P. (2004). Technical design of condition based maintenance system - a case study using sound analysis and case-based reasoning. In Proceedings of the Maintenance and reliability Conference (pp. 403-410).

Cafagna, D. (2007). Fractional calculus: A mathematical tool from the past for present engineers. IEEE Industrial Electronics Magazine, 101,35-40.

Cover, T., \& Hart, P. (1967). Nearest neighbor pattern classification. IEEE Transactions on Information Theory, 13(1), 21-27.

Duvar, A., Eldem, V., \& Saravanan, N. (1990). A system identification approach for failure diagnosis and detection. In Pro- ceedings of the IEEE International Workshop on Intelligent Motion Control (August, pp. 61-64). IEEE.

Endrenyi, J., Aboresheid, S., Allan, R.N., Anders, G.J., Asgarpoor, S., Billinton, R., ... Singh, C. (2001). The present status of maintenance strategies and the impact of maintenance on reliability. IEEE Transactions on Power Systems, 16, 638-646.

Espindola, J.J.D., Bavastri, C.A., \& Lopes, E.M.D.O. (2008). Design of optimum systems of viscoelastic vibration absorbers for a given material based on the fractional calculus model. Journal of Vibration and Control, 14, 1607-1630.

Funk, P., \& Jackson, M. (2005). Experience based diagnosis and condition based maintenance within production systems. International Congress and Exhibition on Condition Monitoring and Diagnostic Engineering Management, 18. 7 pages.

Gonc salves, L.F. (2011). Desenvolvimento de um sistema de manutenc, a o inteligente embarcado [Implementation of an embeded intelligent maintenance system]. Porto Alegre: Universidade Federal do Rio Grande doSul.

Gorenflo, R., \& Mainardi, F. (2008). Fractional calculus: Integral and differential equations of fractional order. In A. Carpinteri and F. Mainardi (Eds.), Fractals and Fractional Calculus in Continuum Mechanics (pp. 223-276). New York, NY: Springer Verlag.

Gutie'rrez-Carvajal, R.E., Rosário, J.M., \& Machado, J.T. (2010). Fractional order calculus: Basic concepts and engineering applications. Mathematical Problems in Engineering, 2010, 375858, 19 pages.

Hartley, T.T., \& Lorenzo, C.F. (2003). Fractional-order system identification based on continuous order-distributions. Signal Processing, 83, 2287-2300.

Jayaswal, P., Wadhwani, A.K., \& Mulchandani, K.B. (2008). Machine fault signature analysis. International Journal of Rotating Machinery. 2008, 10 pages.

Kantardzic, M. (2003). Data mining concepts, models, methods, and algorithms. NJ, USA: IEEE.

Kantardzic, M. (2011). Data mining: Concepts, models, methods, and algorithms (2nd ed. ). NJ, USA: John Wiley \& Sons.

Lagarias, J.C., Reeds, J.A., Wright, M.H., \& Wright, P.E. (1998). Convergence properties of the nelder-mead simplex method in low dimensions. SIAM Journal of Optimization, 9(1), 112147.

Liu, B., \& Makis, V. (2008). Gearbox failure diagnosis based on vector autoregressive modelling of vibration data and dynamic principal component analysis. IMA Journal of Management Mathematics Advance, 19(1), 39-50.

Ljung, L. (1987). System identification, theory for de user. London, UK: University of Linkoping, Prentice Hall International.

Ma, C., \& Hori, Y. (2004). Fractional order control and its application of $\mathrm{PI}^{\alpha} \mathrm{D}$ controller for robust two-inertia speed control. The 4th International Power Electronics and Motion Control Conference IPEMC, 3, 1477-1482.

Machado, J.T. (2013). Fractional order modelling of dynamic backlash. Journal of Mechatronics, 27(7), 741-745.

Machado, J.T., Galhano, A.M., \& Trujillo, J.J. (2014). On development of fractional calculus during the last fifty years. Scientometrics, 98(1), 577-582.

Machado, J.A.T. (2003). A probabilistic interpretation of the fractional-order differentiation. Fractional Calculus and applied Analysis, 6, 73-80.

Magin, R., \& Ovadia, M. (2008). Modeling the cardiac tissue electrode interface using fractional calculus. Journal of Vibration and Control, 14(9-10), 1431-1442.

Moshrefi-Torbaty, M., \& Hammond, J. (1998). Physical and geometrical interpretation of fractional operators. Journal of Franklin Institute, 335B, 1077-1086. 
Ortigueira, M., Machado, J., \& da Costa, J. (2005, December). Which differ integration? Vision, Image and Signal Processing, IEE Proceedings, 152(6), 846-850.

Petras, I. (2006, July). A note on the fractional-order cellular neural networks. In International Joint Conference on Neural Networks (July, pp. 2012-1024). Vancouver, BC, Canada: Sheraton Vancouver Wall Centre Hotel.

Podlubny, I. (1994). The Laplace transform method for linear differential equations of the fractional order (1st ed. ). Slovak: Slovak Academy of Sciences Institute of Experimental Physics.

Podlubny, I. (2002). Geometric and physical interpretation of fractional integration and fractional differentiation. Fractional Calculus and Applied Analisis, 5(4), 367386.

Santos, F.M.D.C., Silva, I.N.D., \& Suetake, M. (2012). Sobre a aplicaplicac,ão de sistemas inteligentes para diagnóstico de falhas em máquinas de induc áo - uma visc,ão geral [About the application of intelligent systems for failure diagnosis in induction machines-A general insight]. Sba Controle \& Automac, ão, 23(5), 553-569.

Shikari, B., \& Sadiwala, C. (2004). Automation in condition based maintenance using vibration analysis (Technical report). Bhopal: Maulana Azad National Institute of Technology.

Valério, D., Trujillo, J.J., Rivero, M., Machado, J.T., \& Baleanu, D. (2013). Fractional calculus: A survey of useful formulas. The European Physical Journal, 222(8), 1827-1846.

Vinagre, B.M. (2007). Optimal fractional controllers for rational order systems: A special case of the Wiener-Hopf spectral factorization method. IEEE Transactions on Automatic Control, 52(12), 1078-1086.

Wang, D., Wang, X., \& Han, P. (2010). Identification of thermal process using fractional-order transfer function based on intelligent optimization. In IEEE/ASME International Conference on Mechatronics and Embedded Systems and Applications (MESA) (Vol. 1, pp. 498-503). Qingdao, ShanDong, China: IEEE. 